\title{
Radiological Image of the Tyrosine Kinase Inhibitors Response of Metastatic Renal Cell Carcinoma
}

\author{
Laura Cerón*, Juan Carlos Aparicio, Victoria Garriga, Sara Cros, Ana Narbona and Jorge Hernández \\ Radiology Department, Hospital General de Granollers, Spain
}

Submission: April 16, 2018; Published: April 25, 2018

"Correspondence Address: Laura Cerón Nasarre, Radiologist, Resident of Radiology Department, Hospital General de Granollers, Barcelona, Spain, Email: lceron@fhag.es

\begin{abstract}
Renal cell carcinoma (RCC) represents $2-3 \%$ of adult malignant tumors and constitutes $90 \%$ of the malignant tumors that affect the kidney. RCC metastatic disease (m-RCC) occurs in a significant percentage and is usually associated with an overall poor prognosis. This tumor is hypervascular and that is why tyrosine kinase inhibitors (TKI), that target the intracellular kinase domain of the vascular endothelial growth factor receptor, have become established as the most successful drug in this disease. These drugs interfere in the angiogenesis of the RCC, thus modifying the radiological image by CT with contrast, making them necessary to study the evolution of the radiological aspect of the findings. Because of mechanism of action of TKI is complex and its effect is different according to the organ in which they act, a heterogeneous radiological response of the metastatic lesions can be expected. Knowledge of this different behavior depending on the organ is mandatory to correctly evaluate the response to treatment.
\end{abstract}

Keywords: Metastatic renal cell carcinoma; Antiangiogenic therapy; Radiological response; Intratumour heterogeneity; Tyrosine kinase inhibitors; Functional imaging

Abbreviations: RCC: Renal Cell Carcinoma; TKI: Tyrosine Kinase Inhibitors; VEGF: Vascular Endothelial Growth Factor; HIF: Hypoxia-Inducible Factor; ADC: Apparent Diffusion Coefficient; CT: Computerized Tomography

\section{Introduction}

Renal cell cancer (RCC) represents $2-3 \%$ of adult malignant tumors and constitutes $90 \%$ of the malignant tumors that affect the kidney. It is considered that one third of patients will present either with locally advanced tumor or with metastases. In addition, another third of patients may develop metastatic disease after initial treatment. M-RCC disease occurs in a significant percentage and is usually associated with an overall poor prognosis, being currently $2 \%$ mortality.

Traditionally, RCC has been remarkably resistant to conventional chemotherapy and radiotherapy, which also justifies a worse prognosis. As it is already known, RCC is a hypervascular tumor due to the increased transcription of vascular endothelial growth factor (VEGF) regulatory genes that occurs in its cells, which leads to an increase in angiogenesis. This is due to the intracellular accumulation in hypoxia-inducible factor (HIF). In turn, the increased intracellular HIF is due to the inactivation by mutation or loss of expression of the protein transcript of the Von Hippel Lindau gene (pVHL). While HIF is mostly active in hypoxic conditions, VHL-defective RCC shows constitutive activation of HIF even in oxygenated environments, which induces the expression of a number of angiogenesisrelated factors (Table 1).

Table 1: Relationship between transduction pathways and specific biologic targets for antiangiogenic therapies in renal cell carcinoma.

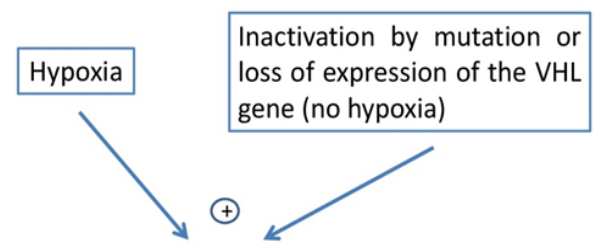

Intracellular accumulation of HIF

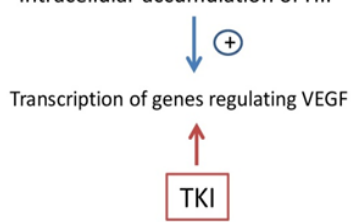

The emergence of new drugs designed to target and interfere with specific aberrant biological pathways have become mandatory. In this way, TKI drugs, that targets the intracellular kinase domain of the vascular endothelial growth factor receptor, have become established as the most successful drug in 


\section{Cancer Therapy \& Oncology International Journal}

this disease. Antiangiogenic-targeted therapies are significantly active in patients with metastatic RCC. Overproduction of VEGF is a key feature in this type of tumors, which results in the activation of angiogenesis that explains the hypervascular nature of RCC (Figure 1).

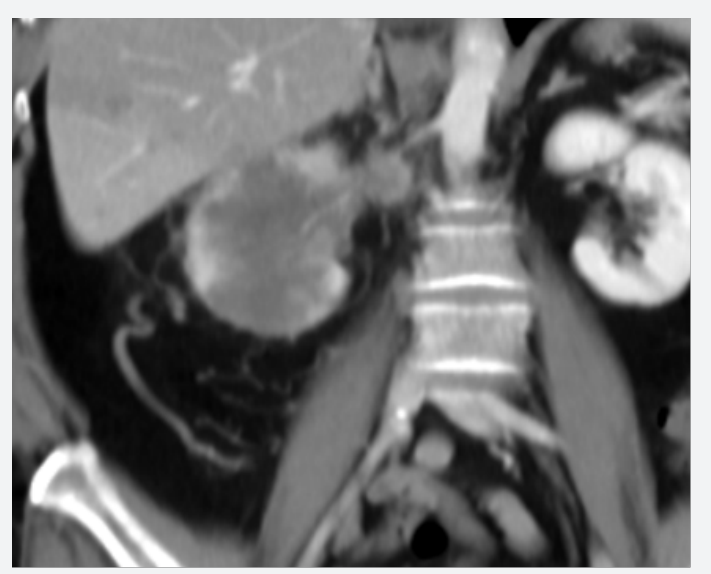

Figure 1: CT study with intravenous contrast. Increased vascularization in right perirenal fat is identified due to an increase in angiogenesis with origin in iliac vessels.

Four main histological subtypes of RCC have been described: clear cell $(75 \%)$, papillary (15\%), chromophobe $(5 \%)$, and oncocytoma (5\%). These histological subtypes also have implications for prognosis and treatment response. Clear cell RCC shows a worse prognosis than papillary or chromophobe tumors and responds very well to antiangiogenic agents, while response of papillary RCC to these agents is limited. For the treatment with TKI, all patients provided signed written informed consent. Our hospital used the standard scheme of Sunitinib and radiological assessment was performed the ended of three cycles, following with CT each three cycles, in case of response.

It has been seen that the radiological response of RCC metastases to these drugs is heterogeneous according to the affected organ [1]. This could be due to differences in vascularization between organs and the molecular intratumour heterogeneity that in the RCC is considered up to $56 \%$. Antiangiogenic agents often cause decreased tumor vascularization and necrosis, leading to a reduction in the attenuation and enhancement of the lesions, which should be determined by the implementation of these criteria. Additionally, functional imaging techniques seem to be sensitive methods for detecting clinically relevant antiangiogenic druginduced modifications in metastatic RCC. Among them, dynamic contrast enhanced (DCE) imaging techniques, such as perfusion computed tomography (CT), DCE-magnetic resonance imaging (DCE-MRI), and DCE-ultrasound (DCE-US) it seems reasonable to think that they could be the most appropriate techniques to predict the response to TKI in these patients.

Perfusion CT is a feasible technique to assess tissue perfusion parameters in patients with metastatic RCC, which correlate positively with microvascular density and may reflect angiogenesis of renal cancer. Diffusion weighted-MRI (DW-MRI) may show apparent diffusion coefficient (ADC) changes. An increased $A D C$ values have been described with antiangiogenic therapies if there is significant tumor necrosis. With the treatment with TKI it is considered that disease stabilization should be regarded as a treatment benefit. In line with this, patients with metastatic RCC presenting disease symptoms may demonstrate clinical improvement after antiangiogenic therapy initiation, even if no response according to RECIST has been demonstrated. In these patients, clinical improvement should be regarded as a clinical response to treatment. Based on all these considerations, experts agree that improvements in quality of life, and secondary in progression-free survival, are the main objectives of treatment administration in these patients.

With the introduction of these new drugs, the question arises as to how these patients should be followed up. As a general rule, an imaging evaluation should be done prior to treatment initiation. Subsequent evaluations, every 3 months during the first year, should be performed in the thorax, abdomen, and pelvic regions, as well as additional assessments in brain, skeleton, etc. as required, depending on the symptoms of each individual patient [2]. After first year treatment imaging evaluation should be done every 6 months if the patient remains clinically stable. However, no definite frequency and duration of the whole evaluation process have been established for patients with metastatic RCC.

To avoid possible biases when evaluating the radiology response, it may be convenient to establish some parameters. The absence of standardization can involve aspects such as the phase of enhancement (arterial or portal) evaluated using the CT scan, volume and flow of the contrast injected to the patient for the correct imaging evaluation, poor selection of target lesions during the baseline evaluation, and inaccurate measurements of these lesions. Therefore, a thorough approach is essential in each tumor response evaluation in order to avoid bias and obtain accurate results [3].

The metastasis of RCC has some specific features that are important to consider to achieve correct diagnosis and cancer staging and to detect recurrence. RCC can grow to a large size before they become clinically evident because of their retroperitoneal location and the rich vascular supply of the kidneys. RCC metastasizes in three basic ways: by continuity, hematogenous and lymphatic dissemination. RCC typically metastasizes to the lung, bone, lymph nodes, liver, adrenal glands, and brain, although practically any organ may be affected. $61 \%$ of patients with RCC have metastatic disease in a single site, and 39\% have metastases in multiple sites. Patients with lung-only metastases seem to present a better survival than patients with other sites, whereas liver and bone metastases are associated with a worst prognosis. The regional lymph nodes in RCC are the renal hilar and retroperitoneal nodes (paraaortic and paracaval), which are most commonly affected. 


\section{Cancer Therapy \& Oncology International Journal}

\section{Pulmonary Metastases}

Account for $45 \%$ of all metastases from renal cell carcinoma, thus representing the most common anatomic site of disseminated RCC. Pulmonary metastases are usually asymptomatic ( $90 \%$ of cases). On CT images, they manifest as multiple nodules $(75 \%$ of cases) or solitary nodules $(25 \%)$. Lesions are usually well circumscribed, round or oval, smaller than $2 \mathrm{~cm}$ and in sub $\neg$ pleural locations. Patients with lung-only metastases seem to present a better survival than patients with other sites (Figure 2).

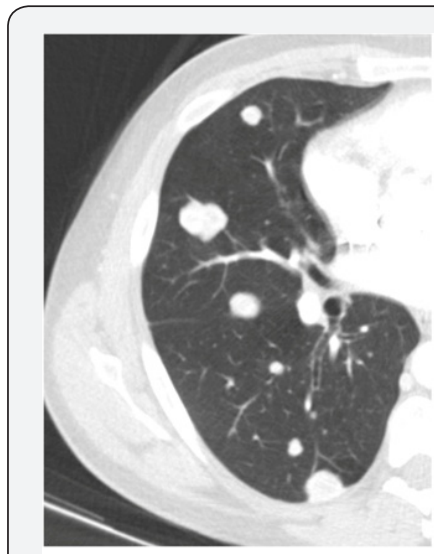

a.

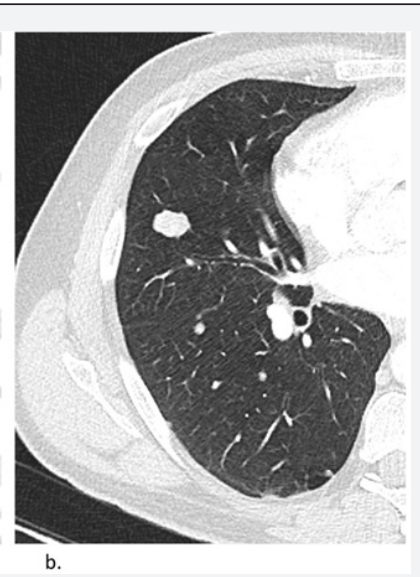

Figure 2: Pulmonary metastases of RCC.

a) Axial CT image of the lung shows multiples solid nodules located in the right superior and inferior lobes, the biggest one measures $2.6 \mathrm{~cm}$.

b) Axial CT image of the lung shows reduction of all pulmonary lesions. Although, smaller metastases are also seen.

\section{Bone Metastases}

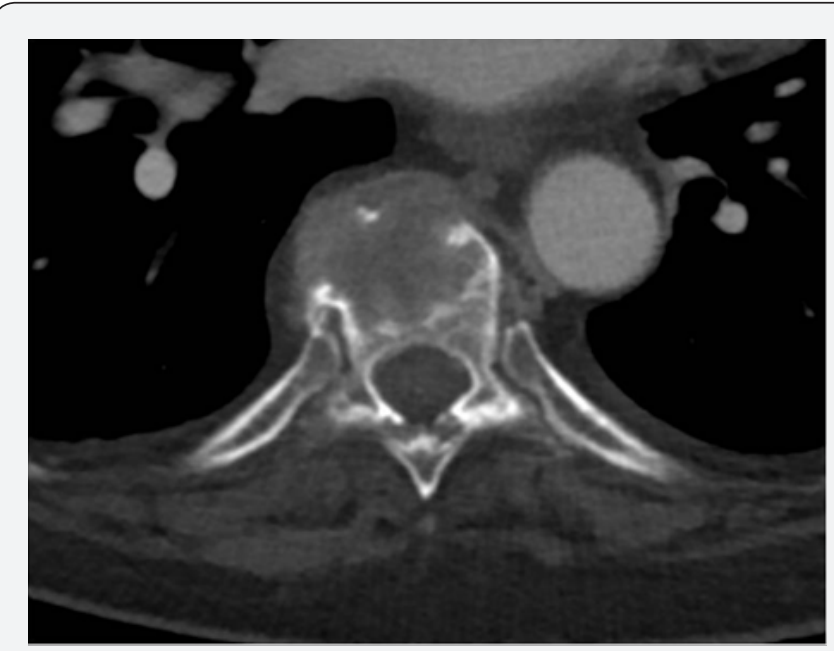

Figure 3: Bone metastases of RCC. Axial abdominal CT image obtained without contrast material administration shows a large osteolytic bone lesion with an associated soft-tissue component involving the T3 vertebral body.

Appear in $30 \%$ of all patients with metastatic RCC, representing the second most common site of distant disease. Frequently, are associated with severe co morbidity, causing pain and pathologic fractures with subsequent functional impairment.

Bone metastases in RCC are mainly osteolytic (hypovascular), related to excessive activation of osteoclasts. Most commonly lo $\neg$ cated in the axial skeleton; usually the vertebral bodies are involved more often than the pedicles. These lesions are usually extremely destructive and may be associated with an enhancing soft-tissue component (Figure 3).

\section{Lymph Node Metastases}

Lymph node metastases are the third most common group of metastatic RCC lesions. Diagnosis of lymph node involvement is based on morpho $\neg$ logic criteria, especially size increase seen at CT. It is generally accepted that abnormal lymph node has a maximum short-axis diameter of minimum $10 \mathrm{~mm}$. The lesions show heterogeneous contrast hyper enhancement and some show small areas of central necrosis (Figure 4).

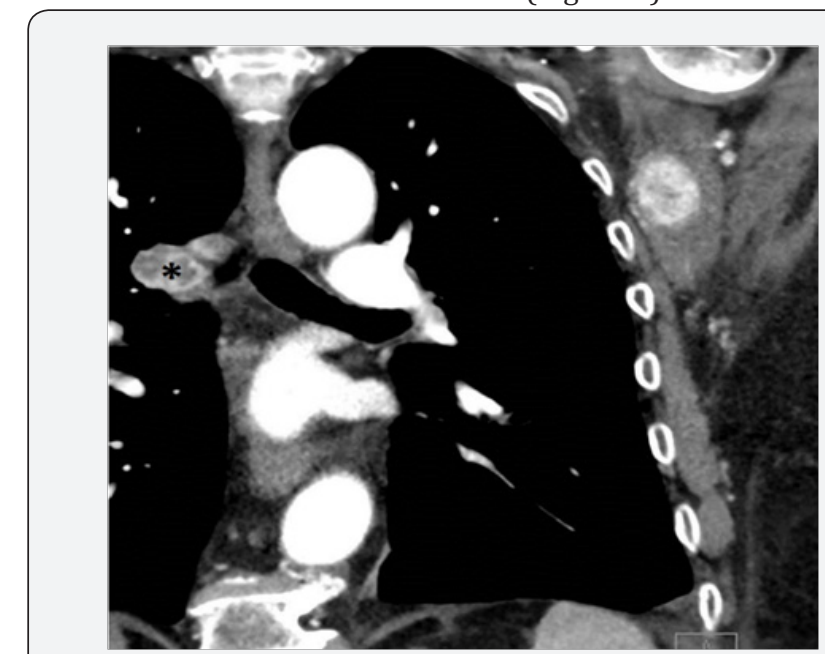

Figure 4: Subscapularis muscle and lymph nodes RCC metastases. Coronal arterial phase CT image shows a hypervascular well-defined round lesion located in the left subscapularis muscle. In the same image, it can also be observed multiple enlarged lymph nodes with a heterogeneous contrast hyper enhancement and some show small areas of central necrosis $\left({ }^{*}\right)$.

Liver Metastases

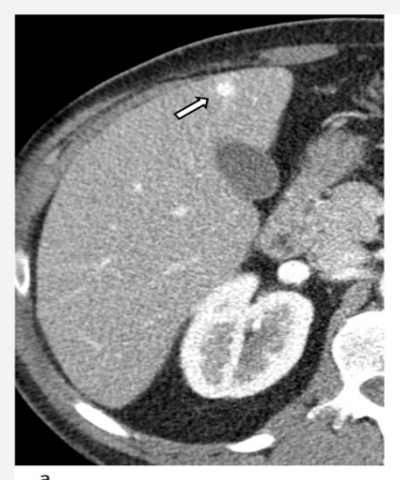

Figure 5: Liver metastasis of RCC.

a. Axial arterial phase CT image shows hypervascular lesion (arrow) compatible with M1 disease.

b. Axial portal phase CT image shows two hypovascular lesions also compatible with M1 disease. 


\section{Cancer Therapy \& Oncology International Journal}

$20 \%$ of patients with RCC develop liver metastases. These lesions usually appear as ill-defined low attenuation lesions that may show peripheral enhancement or appear as hypervascular masses with or without central necrosis (Figure 5).

\section{Brain Metastases}

Appear in eight percent of all patients with disseminated RCC. These patients are often asymptomatic (90\% of cases). Brain metastases appear as enhancing nodules with associated vasogenic edema and usually mani-fests as a solitary lesion with a hemispheric locaᄀtion (Figure 6).
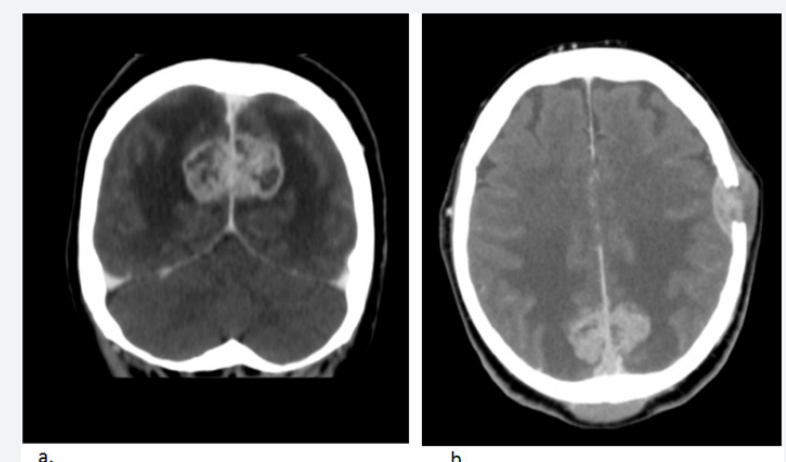

igure 6: Brain metastasis of RCC

a) Coronal CT section with administration of intravenous contrast in which a dural lesion is observed in the cerebral sickle that grows towards both parietal lobes, with peripheral enhancement and important perilesional vasogenic edema.

b) Axial CT section with intravenous contrast administration of the same patient, in addition to the anterior lesion, two welldefined osteolytic lesions with soft tissue mass, one in the middle line parieto-occipital and another one on the left frontoparietal bone.

Uncommon location of RCC Metastases

\section{Adrenal metastases}

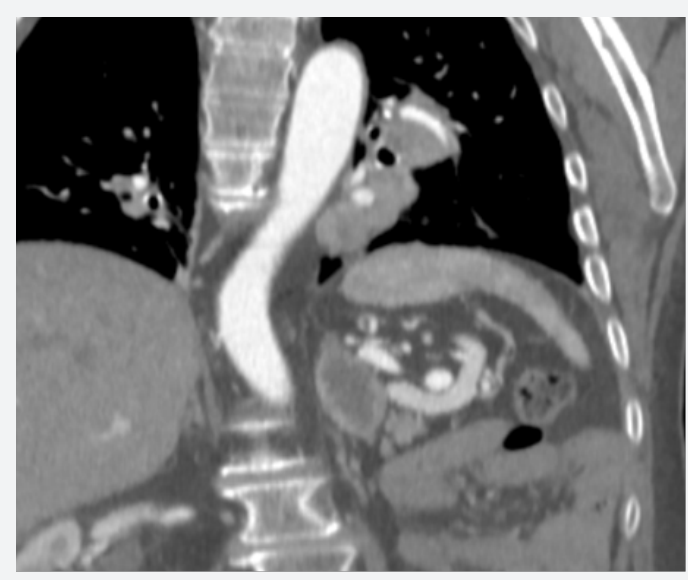

Figure 7: Adrenal metastases of RCC. Coronal venous phase CT image shows a left adrenal lesion. After intravenous contrast administration, this lesion has a heterogeneous enhancement, suggestive of malignancy.

These lesions are rare, develop ing in only $9 \%$ of patients with RCC, and are usually functionally silent. They are observed like hypervascular lesions in adrenal glands (Figure 7).

\section{Gastrointestinal tract metastases}

It is a very rare location of the RCC metastasis but in our hospital we have had a case with a gastric metastasis. The lesion behaved as a hypervascular round mass (Figure 8).

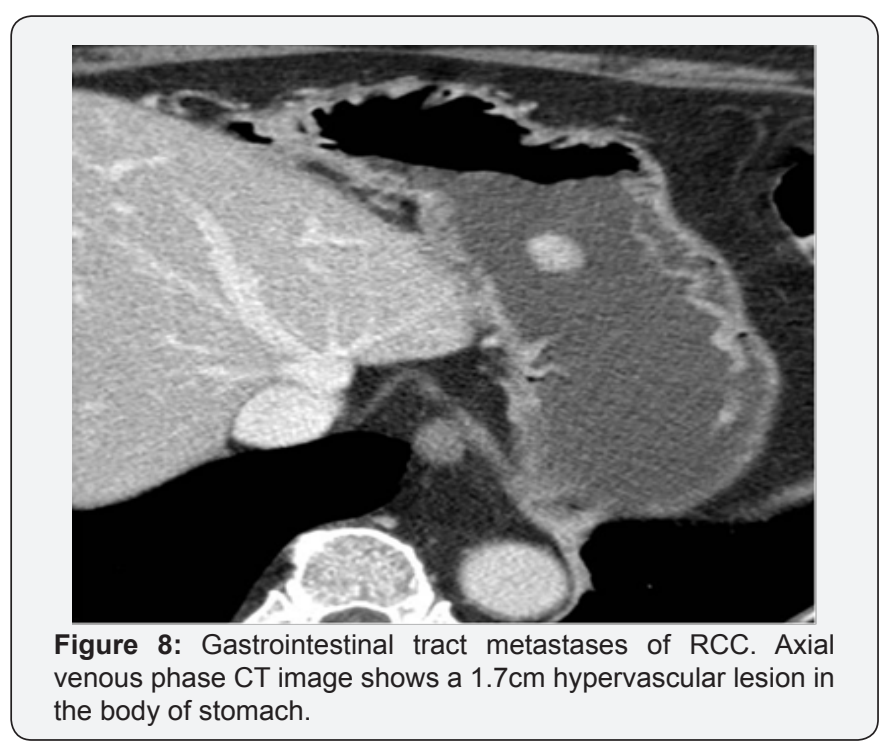

\section{Muscle metastases}

There are not many reported cases of metastatic RCC with muscle injuries. However, among our patients.

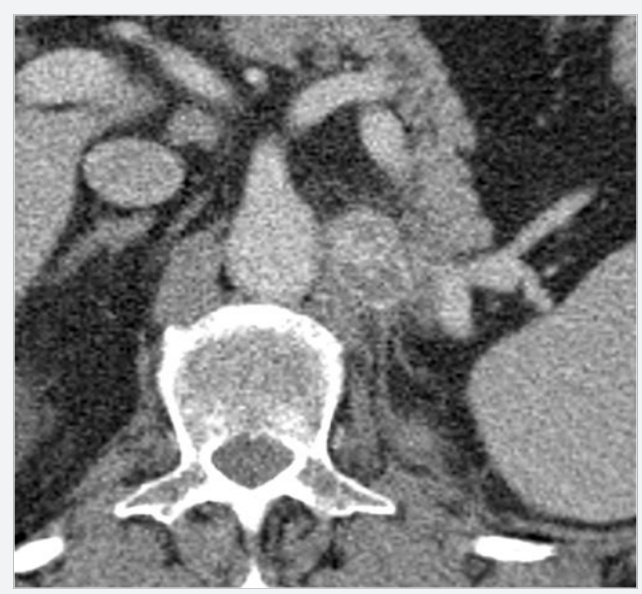

Figure 9: Muscle metastases of RCC. Axial venous phase CT image shows a $2.8 \mathrm{~cm}$ lesion in the infiltrating left psoas muscle with a peripheral enhancement after contrast administration.

We have been able to observe several cases, both at the locoregional level, with the psoas muscle being the most common location, as well as at a distance, in the subscapularis muscle. Also, we can see a diaphragm one. They behave like rounded hypervascular lesions with peripheral enhancement. (Figure 4), (Figures 9 \& 10).

\section{Peritoneal and Retroperitoneal Implants}

They appear as nodular lesions and in most cases they are multiple and can converge. These masses have a hypervascular 


\section{Cancer Therapy \& Oncology International Journal}

behavior with the administration of contrast and their most frequent location is the perirenal fascia, manifesting itself as a locoregional infiltration (Figure 11).

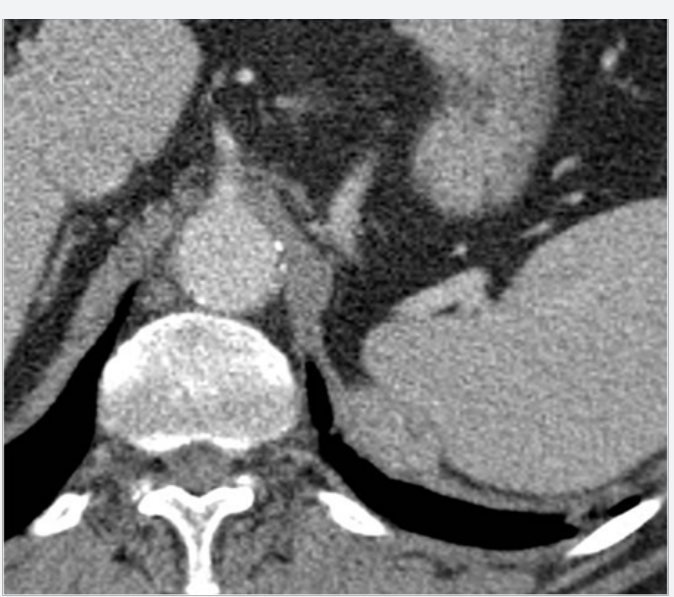

Figure 10: Diaphragm metastases of RCC. Axial venous phase CT image shows a $3.5 \mathrm{~cm}$ lesion located in the left diaphragm with a slight enhancement contrast.

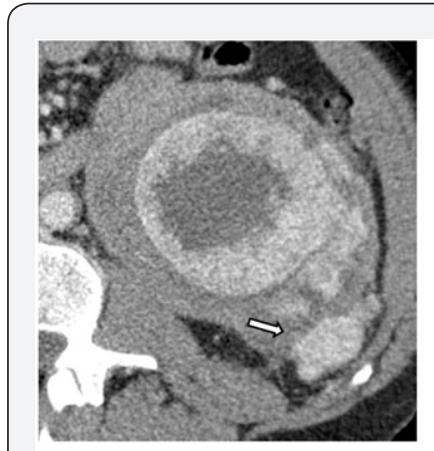

a.

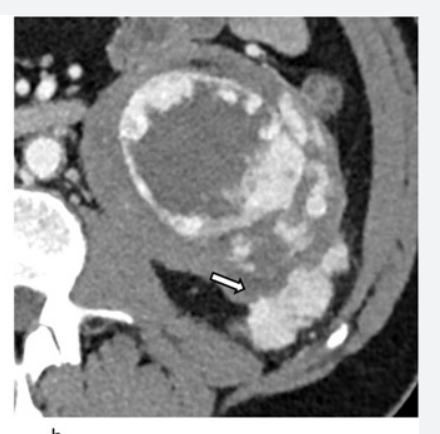

b.
Figure 11: Retroperitoneal metastases of RCC.

a) Axial portal phase CT image shows a solid hyper enhancing nodule in the peritoneum $(4.1 \mathrm{~cm} ; 145.5 \mathrm{UH})$ done prior to treatment initiation.

b) Axial portal phase CT image from the same patient after four months of treatment shows increase in size and enhancement $(5.1 \mathrm{~cm} ; 158 \mathrm{UH})$.

\section{Metastasis Behavior After TKI's Treatment}

Tyrosine kinase inhibitors induce early and extensive necrosis yet without a significant decrease in tumor size. These considerations suggest that standard evaluation criteria based on tumor size, such as RECIST, may not be the best for evaluating the effectiveness of targeted drugs in patients with metastatic RCC because they suggest apparently low response rates that do not reflect the real state of the disease and, consequently, the benefit in terms of time to progression.

Other criteria have been proposed to evaluate the response to these antiangiogenic drugs as changes in lesion size, attenuation and internal structure. Indeed, the importance of marked decreased attenuation (a decreased in attenuation of $40 \mathrm{HU}$ or greater in one or more than one predominantly solid enhancing lesions, evaluated on routine portal venous phase), marked central necrosis ( $>50 \%)$, and size changes depict an early tumor response. On the contrary, the appearance of new metastases and a new marked central filling (new enhancement in a previously nonenhancing mass with homogeneous low attenuation) show a tumor progression.

\section{Conclusion}

Metastatic renal cell carcinoma is a tumor with a very heterogeneous response of its metastases to treatment with Tyrosine kinase inhibitors. It has been seen that the radiological response of RCC metastases to these drugs is heterogeneous according to the affected organ. This could be due to molecular intratumour heterogeneity and probably the differences between vascularization of the different organs. It is necessary for radiologists to know that the metastatic RCC, due to its heterogeneity, can metastasize in any organ, even in the least frequent locations and with atypical behaviors, even after several treatment cycles.

Because these drugs induce early and extensive necrosis yet without a significant decrease in tumor size, it is possible to think that other radiological tests, beyond those that are merely morphological such as RECIST, may be necessary for these patients. Novel functional and molecular imaging techniques may be useful tools in the evaluation of patients with metastatic RCC like dynamic contrast enhanced imaging techniques. One of the main negative aspects associated with these techniques is the pending issue of their standardization in terms of use and acquisition for the clinical practice. Nowadays, there is not sufficient standardization or scientific evidence to abandon anatomical assessment of tumor burden.

\section{References}

1. Santoni M, Santini D, Massari F, Conti A, Iacovelli R, et al. (2013) Heterogeneous drug target expression as possible basis for different clinical and radiological response to the treatment of primary and metastatic renal cell carcinoma: suggestions from bench to bedside. Cancer Metastasis Rev 33(1): 321-331.

2. Paño Brufau B, Sebastià Cerqueda $C$, Buñesch Villalba L, Salvador Izquierdo R, Mellado González B, et al. (2013) Metastatic Renal Cell Carcinoma: Radiologic Findings and Assessment of Response to Targeted Antiangiogenic Therapy by Using Multidetector CT. Radiographics 33(6): 1691-1716.

3. León L, García-Figueras R, Suárez C, Arjonilla A, Puente J, et al. (2014) Recommendations for the clinical and radiological evaluation of response to treatment in metastatic renal cell cancer. Target Oncol 9(1): 9-24. 
Your next submission with Juniper Publishers will reach you the below assets

- Quality Editorial service

- Swift Peer Review

- Reprints availability

- E-prints Service

- Manuscript Podcast for convenient understanding

- Global attainment for your research

- Manuscript accessibility in different formats ( Pdf, E-pub, Full Text, Audio)

- Unceasing customer service

Track the below URL for one-step submission https://juniperpublishers.com/online-submission.php 